\title{
Black Printed Circuit Board-based Micro-Polymerase Chain ReactionChip Structure for Fluorescence Detection Test
}

\author{
Ji-Soo Hwang ${ }^{1,3}$, Jong-Dae Kim ${ }^{2,3}$, Yu-Seop Kim ${ }^{2,3}$, Hye-Jeong Song ${ }^{2,3}$ and Chan- \\ Young Park Pa* $^{2,3}$ \\ ${ }^{1}$ Dept. of Computer Engineering, Hallym University, Korea \\ ${ }^{2}$ Dept. of Convergence Software, Hallym University, Korea \\ ${ }^{3}$ Bio-IT Research Center, Hallym University, Korea \\ \{seattle,kimjd,yskim01, hjsong,cypark\}@hallym.ac.kr
}

\begin{abstract}
This paper proposes the optimal structure of a Printed Circuit Board-based micro-PCR Chip constructed on a PCB substrate using commercial adhesive tapes and plastic films. The proposed micro-PCR chip was composed of four layers. In a previous experiment, the chip had been made with a green $P C B$, yielding an unwanted reflection that prevented accurate analysis. In this study, the solder mask of the PCB substrate was coated black for a higher signal-to-noise ratio, and the area where the reaction chamber was attached was legend-printed with white silk for better reflection of the fluorescence light. The top cover, which was $180 \mu \mathrm{m}$ thick, was also colored black to minimize the noise during fluorescence detection. As a result of carrying out PCR, it was found that the fluorescence brightness increased with increasing numbers of cycles.
\end{abstract}

Keywords: Micro-PCR chip, Double-sided tape, Polymerase Chain Reaction, Acrylic adhesive, Microfluidic channel, Fluorescence Detection Test, Black PCB

\section{Introduction}

A variety of inexpensive and disposable lab-on-a-chip (LOC) devices are being developed for miniaturization, integration, and automation and for point-of-care diagnosis of routine biochemical process $[1,2]$. LOC devices are used for a variety of applications in biotechnology, medical diagnosis and treatment, and basic research [3, 4]. The most important characteristics of LOC devices are their ability to stably process body fluids of cells and aqueous solutions of biomolecules, their cost-effectiveness, and their ability to process smaller amounts of samples [1-2, 5-7]. Because of these requirements, development of microfluidic channels has been actively carried out to process such small amounts. To fabricate these channel, materials such silicon, polymers, and glass have been integrated through etching, baking, and bonding processes that are expensive and difficult $[1,2]$. However, more efficient and inexpensive channel fabrication is possible if recently developed thin and flexible tape is used [2].

Since tapes are mass-produced in various thicknesses, the thermal cycling required for DNA amplification can be effectively performed by reducing the thermal resistance by selecting a thin tape. Also, fabrication is easy because a microfluidic channel is made simply by hollowing out a tape. Moreover, the market for LOC devices is rapidly growing through the mass production of various tapes; even if mass production of the existing method is established, the use of tape is a much less expensive manufacturing method [2].

In a previously reported study, a micro-PCR chip fabricated with double-sided tape showed results similar to those of regular tube-type PCR chips. However, in the regular PCR chip, PCR amplification products can be confirmed at the end point only through the

${ }^{*}$ Corresponding Author 
electrophoresis process; however, there is high risk of contamination in the electrophoresis process. However, the real-time PCR method is a technology that can monitor and analyze the increase of PCR amplification products in real-time. Moreover, its advantage is that accurate quantification of DNA and RNA is possible, allowing quick and easy analysis because electrophoresis is not necessary, and the risk of contamination is low as compared to the conventional PCR method. An experiment was carried out by changing the color of the PCB, the chip substrate, to matte black in order to decrease the signal-to-noise ratio (SNR) that occurs when light is reflected in a PCR chamber during fluorescence detection in the process of implementing a real-time PCR with a biochip. In this study, by conducting the fluorescence detection according to the PCB color, the frequencies of fluorescence brightness are shown. By carrying out a PCR with a new chip structure using a black PCB, the results for PCR performance are shown as a function of the frequency of fluorescence brightness for each cycle. In Section 2, the micro-PCR driving system and black PCB-based structure are discussed, and in Section 3, the results of carrying out brightness experiments using a conventional chip and a PCR cycle with the proposed chip are presented. The conclusion is summarized in Section 4.

\section{Black PCB-Based Micro-PCR Chip}

\subsection{PCR Chip Driving System}

Figure 1shows a block diagram of the control system proposed in this paper. The local system's processor is PIC18F55 (Microchip Technology Inc.) and an analog-to-digital converter (ADC), pulse-width modulation (PWM), and USB interface are included. To heat and cool the PCR chip, the heater and fan are controlled by using the PWM and a field-effect transistor (FET). The thermistor resistance of the PCR chip is changed to a temperature value in digital form, originating from the voltage divider and ADC. USB 2.0 connects the local system and the PC. Every $5 \mathrm{~ms}$, the temperature is recorded in the local system and the value data are sent to the host. At the host, the temperature values are read from the local system and the PWM value is calculated on the basis of a proportionalintegral-derivative (PID) controller mechanism. In other words, at the host, functions related not only to the temperature control but also to PCR protocol execution are processed in the GUI environment through the file input/output.

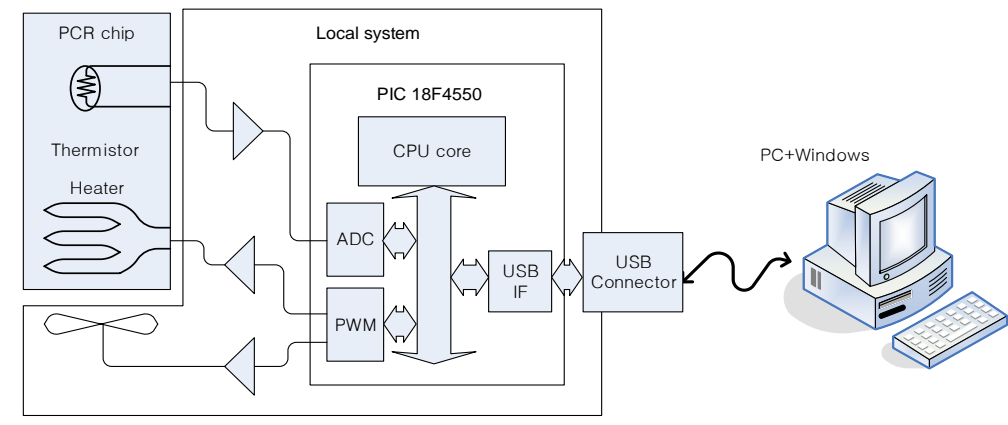

Figure 1. PCR Chip and its Control System

The control system of the PCR chip consists of biochemical functions and user interface functions. The biochemical functions process consists of the protocol and chip's temperature control part. The PCR protocol means carrying out actions set to run at a set temperature for a certain time repetitively. For heating or cooling to a set temperature by sensing the temperature, the chip's temperature control was designed to increase or decrease the temperature by using the heater or the cooling fan. The basic biochemical function was designed and created to allow direct management, editing, and control 
through the user interface. These two functions are integrated in the embedded system as one process. A system of local-host structures through the PC was made as shown in Figure 1 because it was determined to be more efficient to access the embedded system with the user interface on the PC to control it easily. The temperature of the PCR chip was controlled by setting appropriate heating and cooling temperatures with the temperature measurements and periodic control procedure using the heater and fan. The PCR chip's temperature was set to be increased or decreased by approximately $10^{\circ} \mathrm{C} / \mathrm{s}$, and the processing period was set to control the temperature with an error range of less than $0.5^{\circ} \mathrm{C}$ within $50 \mathrm{~ms}$. Since it was difficult to set an appropriate resolution with the normal timer provided by Windows PC, the resolution was set to be less than $1 \mathrm{~ms}$ by using a highprecision event timer (HPET).This was to facilitate the use of various multimedia applications in the future. The overall cost can thus be reduced because the major functions can be performed at the host in the local-host system using a PC [9-12].

\subsection{The Proposed PCR Chip Structure}

The micro-PCR chips proposed in the past were divided into lower and upper PCB parts. At the lower part, a heater and a sensor were attached; the upper PCB part was fabricated to cool or transfer the heat to the chamber through the substrate after attaching a thermal pad; the holes in the cover were divided into inlet/outlet holes and an air hole. However, the micro-PCR chip proposed in this paper consists of a four-layer structure, as shown in Figure 2.At the very bottom, a heating circuit for heating, with the PCB base and a thermal sensor for sensing temperature, are attached [13-15].

To prevent a fluorescence substance from sticking to the PCB, a box tape of PP material was attached. To set the height of the microfluidic channel to $400 \mu \mathrm{m}, 200-\mu \mathrm{m}-$ thick double-sided tape was attached in two layers. The chamber was fabricated by covering it with a $180-\mu \mathrm{m}$-thick PP material cover. In the case of micro-PCR chips reported in the past, PCR was performed by making a microfluidic channel using a regular green $\mathrm{PCB}$, and the PCR performance was verified through electrophoresis.

In the case of implementing a real-time PCR system with the conventional chip structure, when light was received in the fluorescence detector at a green PCB, the light was reflected, which acted as a noise, which reduced the overall signal-to-noise ratio (SNR), which adversely affected the measurement. To prevent the reflection on the parts other than the chamber, after cutting black matte aluminum tape into the shape shown in Figure 2(a), it was attached on the cover. When DDW and PCR were finished, the brightness was compared.

When PCR was performed by fabricating a chip according to the structure of Figure 2, because the black matte aluminum tape attached on the cover conserved heat, it became an obstacle to controlling the temperature, acting sensitively in the process of carrying out PCR. Therefore, the micro-PCR chip proposed in this paper used a non-light-reflective black matte-type PCB. White-silk legend printing was used only on the chamber where the fluorescence detection was performed to easily distinguish the change of fluorescence amount while performing the PCR cycle. 


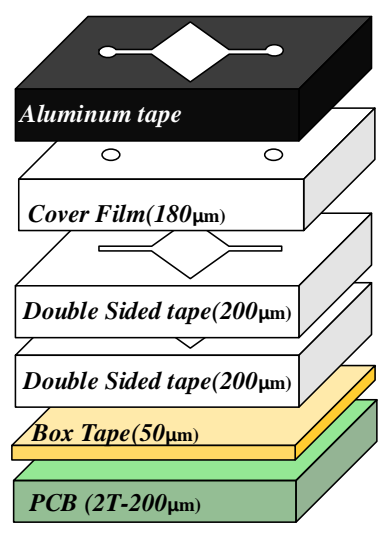

(a) Assembly diagram

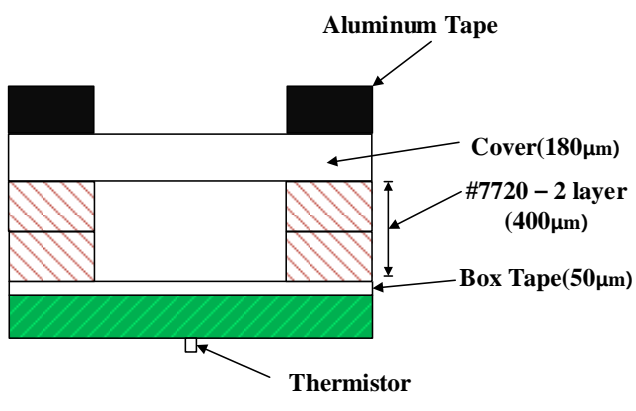

(b) Cross section

Figure 2. Structure of the Micro-PCR Based PCR Chip Used in the Comparison Experiment of Fluorescence Brightness at the Early Stage of Experiment

To prevent heat conservation inside the chip while performing PCR, the cover was colored with a black marker pen, as shown in Figure 3, so that parts other than the chamber could be easily distinguished.

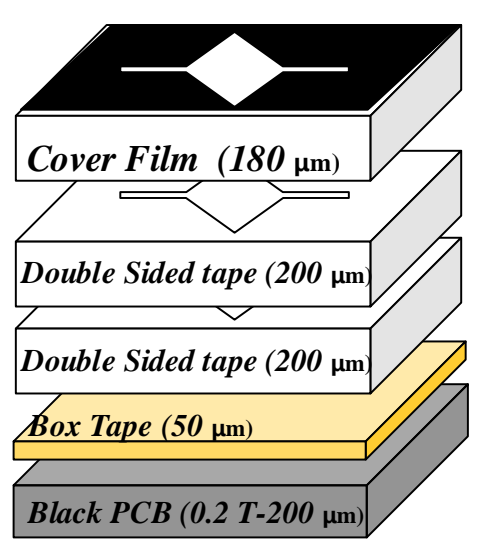

(a) Assembly diagram

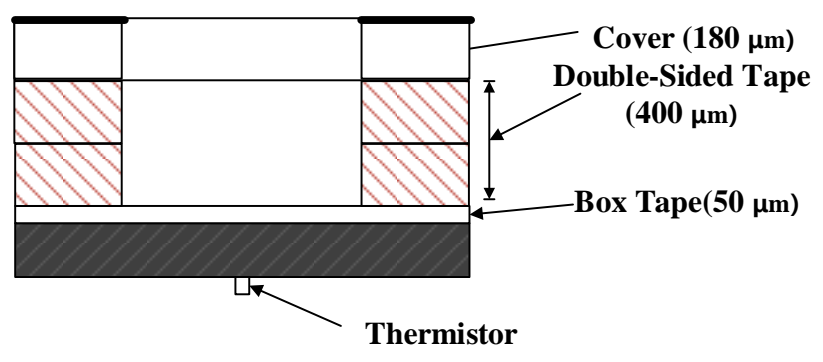

(b) Cross section

Figure 3. Structure of Micro-PCR Chip using a Black PCB

A double-sided tape, which was an important material that acted as the chamber in the micro-PCR chip, was the YT-7720(510) model manufactured by YOUNGWOO (South Korea).As reported in the past, it can withstand high temperatures of $95^{\circ} \mathrm{C}$ and pressure when expanding. The adhesive was acrylic adhesive, and the carrier of the double-sided tape was composed of PET. The structure shown in Figures 2 and 3 is tube-shaped, in which injection of the reagent is facilitated through the inlet/outlet holes on both sides. The chamber in which the PCR was performed in the middle was fabricated by drilling a hole in a diamond shape.

Table 1. Box and Double-Sided Tapes Used in the Experiment

\begin{tabular}{|c|c|c|c|}
\hline Heading level & Manufacturer & Tape Number & Adhesive Type \\
\hline Box Tape & $3 \mathrm{M}$ & \#309 Mini Clear & $\begin{array}{c}\text { Acrylic } \\
\text { Adhesive }\end{array}$ \\
\hline
\end{tabular}




\begin{tabular}{|c|c|c|c|}
\hline Double-Sided Tape & $\begin{array}{c}\text { YoungWoo } \\
\text { (Korea) }\end{array}$ & $\begin{array}{c}\text { YT-7720(510) } \\
\text { (Double-Coated Tape:0.2mm) }\end{array}$ & $\begin{array}{c}\text { High-Adhesion } \\
\text { Acrylic }\end{array}$ \\
\hline
\end{tabular}

\section{Experiments and Results}

Figure 4(a) shows the fluorescence measurement apparatus used in the experiment. Images were captured with DSLR (Canon 1100D), and to detect FAM fluorescence, a blue LED (9600 mcd) was illuminated diagonally. Figure 4(b) shows a micro-PCR chip fabricated with black PCB.As shown schematically in Figure 3, the chip was fabricated into a structure in which an increase or decrease of frequency could easily be observed by printing a white-silk legend on the chamber to make the distinction of fluorescence measurement easy. In the experiment, the PCR process was carried out according to protocol. To carry out temperature control in the proposed system, the PCR process, i.e., the denaturation, renaturation, and extension processes, was carried out. A total of 40 cycles were carried out for $3 \mathrm{~min}$ at $95^{\circ} \mathrm{C}, 15 \mathrm{~s}$ at $95^{\circ} \mathrm{C}$, and $1 \mathrm{~min}$ at $60^{\circ} \mathrm{C}$.

As for the reagent used in the PCR, the experiment was carried out by adding 1 $\mathrm{ng} / 11 \mu \mathrm{L}$ of DNA (Chlamydia trachomatis), $12.5 \mu \mathrm{L}$ of master mix, $10 \mathrm{pM} / 0.5 \mu \mathrm{L}$ of primer F, $10 \mathrm{pM} / 0.5 \mu \mathrm{L}$ of primer R, and $10 \mathrm{pM} / 0.5 \mu \mathrm{L}$ of probe.

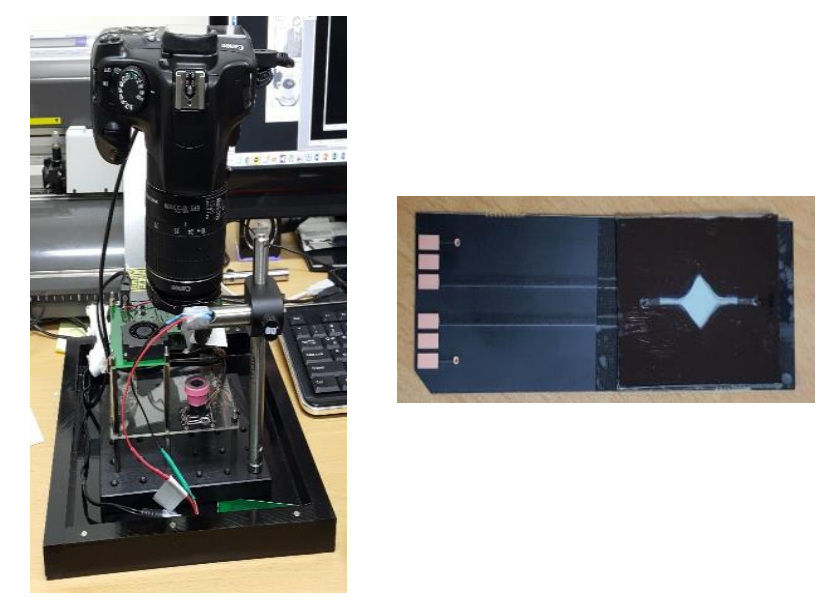

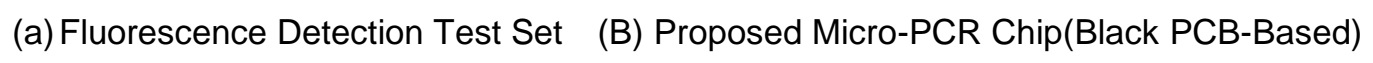

\section{Figure 4. Apparatus used for (A) Fluorescence Measurement And (B) The Black PCB-Based Chip used in the Experiment.}

Figure 5 shows the images captured after performing DDW and PCR in the $400 \mu \mathrm{m}$ chamber by changing the TV value. With the green PCB, there is some reflection, and the differences of fluorescence brightness cannot be easily distinguished. However, the fluorescence brightness of the PCR products of DDW and CT (Chlamydia trachomatis) DNA can be distinguished. 


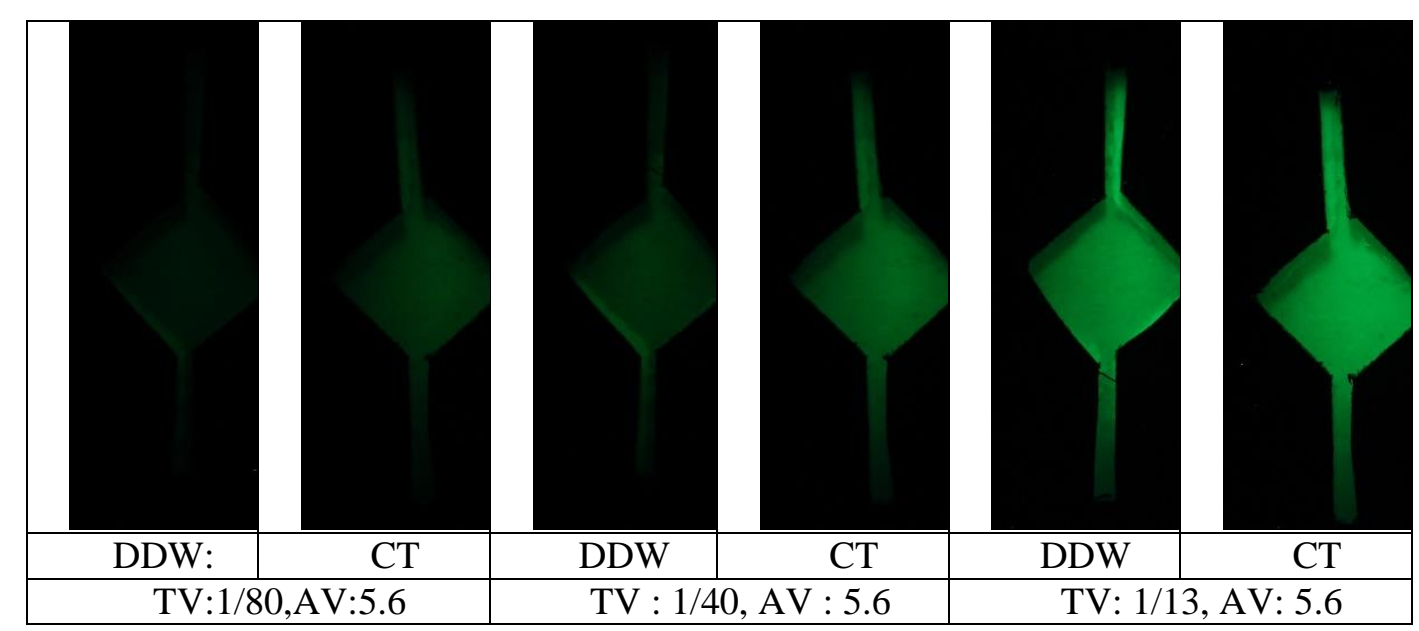

Figure 5. Fluorescence Comparison Experiment Of Chamber Fabricated With Green PCB (DW Vs. Plateau).

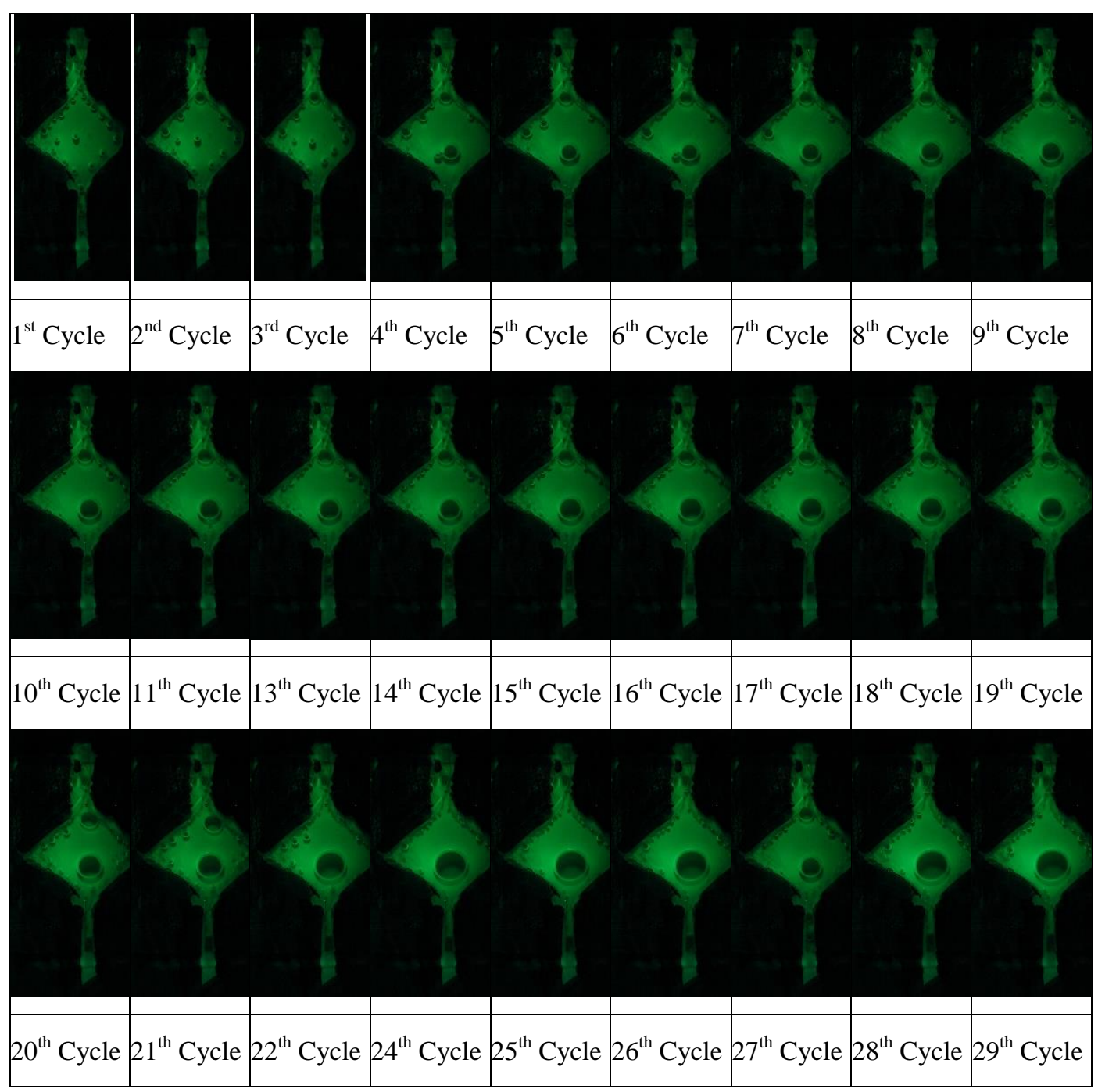



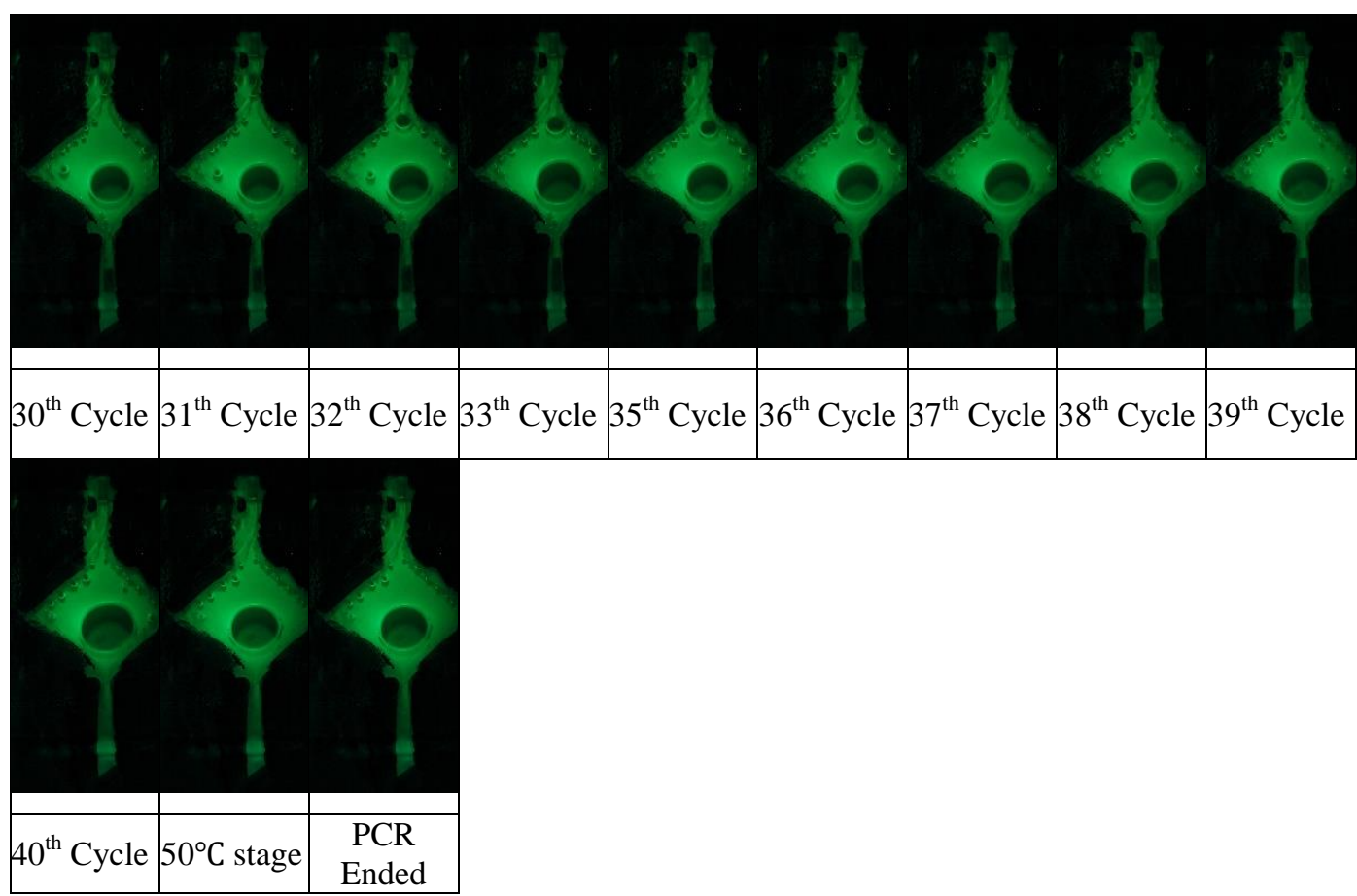

Figure 6. Changes of Fluorescence Brightness by PCR Cycle (Black PCBBased Micro-PCR Chip)

Figure 6 shows photographs of the changes of fluorescence brightness of the PCR cycle performed with the PCR protocol provided above. The experiment was carried out using the fluorescence photography setup shown in Figure 4(a), and the images were captured with DSLR whenever a cycle was proceeded at the stage of $72^{\circ} \mathrm{C}$ (DNA extension state of PCR) which was the middle amplification part in the PCR process excluding the initial stage. Because the images were captured manually, photographs could not be taken for about three cycles. As shown in the figure, it was confirmed that the brightness of fluorescence increased as the PCR progressed.

Figure 7 shows the resulting values by using mean, median, and the Bhattacharyya distance of image brightness according to the PCR cycle. In the $12^{\text {th }}, 23^{\text {rd }}$, and $24^{\text {th }}$ cycles, because there was no image captured, there is no data value and empty spaces are shown; in the other sections, all pertinent values are shown.

The mean and Bhattacharyya distance, excluding the median value, all showed that as the PCR progressed, the brightness values decreased slightly as compared to those before PCR. After the $15^{\text {th }}$ cycle, an exponential increase is shown, but at the $27^{\text {th }}$ cycle, the values sharply decrease. The values also decrease sharply at the $33^{\text {rd }}$ cycle, and at the $35^{\text {th }}$ cycle, a decreasing trend is shown again. After reaching the peaks at the $50^{\circ} \mathrm{C}$ section, a decreasing trend after finishing the PCR is shown.

Furthermore, although the mean and Bhattacharyya distance show similar patterns, the Bhattacharyya distance, expressing the distance to two distributions, shows a more exponential increase. At the latter half of the PCR cycle, the graph shows a saturated form. The brightness of fluorescence can be compared with the mean values of selected areas in the images captured for each cycle; however, because the quantitative brightness is difficult to show with only the mean values, it is also shown with Bhattacharyya distance. The differences of brightness were also distinguished with the Bhattacharyya distance. 


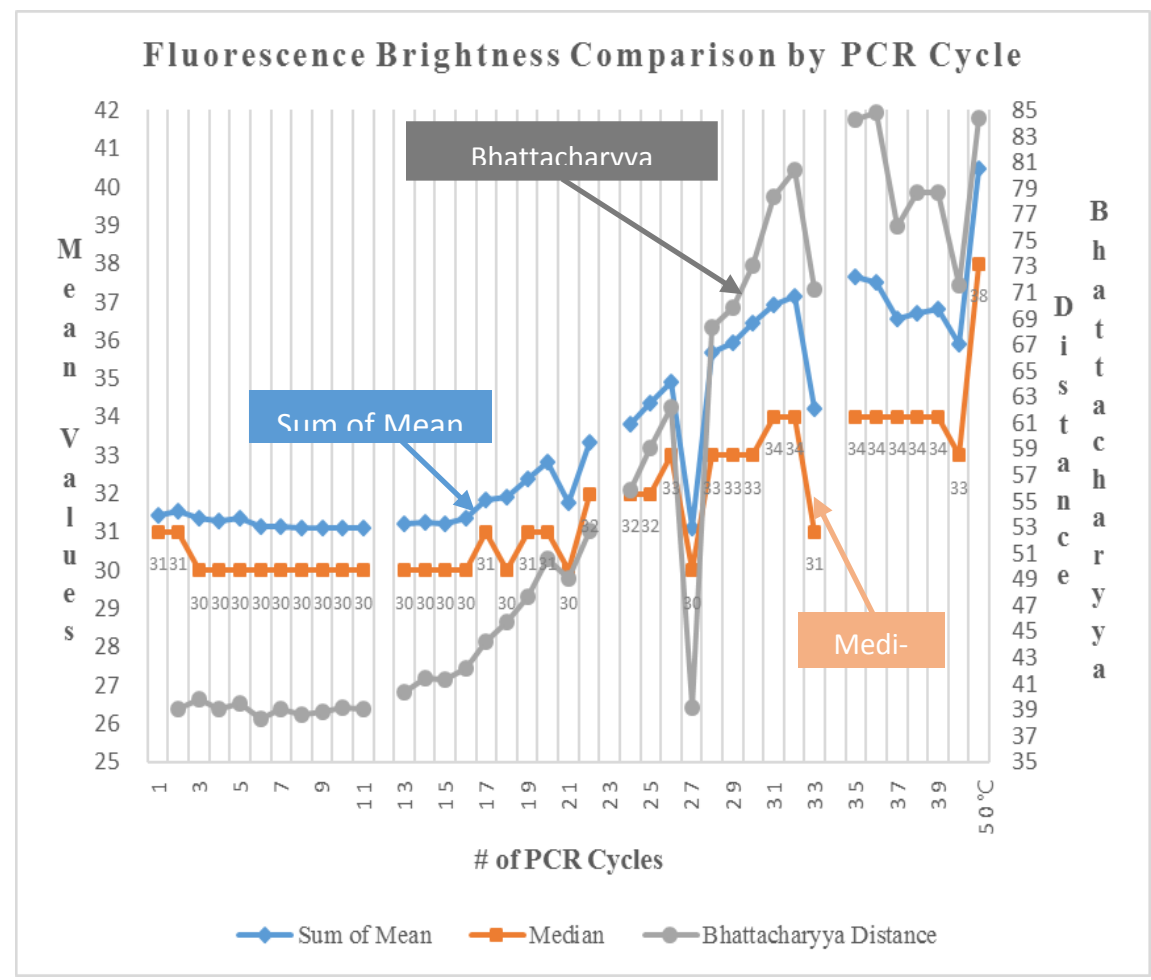

Figure 7. Fluorescence Detection Test By PCR Cycles

\section{Conclusion}

It was reported in past studies that PCR is possible with a micro-PCR chip fabricated by using double-sided tape. It is sufficient to fabricate it with a green PCB, which is frequently used, when carrying out a conventional PCR. However, when carrying out a fluorescence detection test, the blue LED light is reflected by the PCB, which acts as noise and reduces the signal-to-noise ratio (SNR). Therefore, the noise was minimized by changing the color of PCB to matte black.While carrying out real-time PCR, the fluorescence brightness changes could be seen as a function of PCR cycle.

Because it was difficult to make a quantitative comparison by mentioning only the sum of the means, which is used frequently in the process of analyzing fluorescence brightness, the increase of fluorescence was analyzed by using the Bhattacharyya distance and comparing the similarities shown by a histogram of images. The Bhattacharyya distance showed more exponential increases than the mean values, and it was very similar to the curve drawn by the amplified amount of PCR products at the actual real-time PCR. If an exponential increase graph can consistently show real-time PCR results, it seems it would also be possible to carry out a real-time PCR with the proposed chip structure.

\section{Acknowledgments}

The research was supported by the Basic Science Research Program of the National Research Foundation of Korea (NRF), which is funded by the Ministry of Education, Science and Technology (2012R1A1A2040381), and by the Research and Business Development 2013 project of the Ministry of Trade, Industry and Energy (N0000907).

\section{References}

[1] D. Patkoa, Z. Martonfalvic, B. Kovacsa, F. Vonderviszta, M. Kellermayerc, R. Horvatha, Sensors and Actuators B 196, 352 (2014). 
[2] M. Focke, D. Kosse, C. Muller, H. Reinecke, R. Zengerle and F. Stettenab, Lab on a Chip 10, 1365 (2010).

[3] J.J. Ramsden, "Biomedical Surfaces, Artech House", Norwood, MA, (2007).

[4] M. Malmsten, "Biopolymers at Interfaces", Taylor \& Francis, New York, (2003).

[5] C. Zhang and D. Xing, "Nucleic Acids Res", vol. 35, no. 4223, (2007).

[6] C. Zhang, D. Xing, and Y. Li, "Biotechnology Advances 25", 483 (2007).

[7] D.P. Herzog, "IVD technology", (2006).

[8] R. Kodziusa, K. Xiaoc, J. Wud, X. Yid, X. Gongd, I.G. Fouldsb and W. Wen, "Sensors and Actuators B 161", vol. 349, (2012).

[9] C. Plaisant, A. Rose, B. Shneiderman and A.J. Vanniamparampil, "IEEE Software 14", vol. 66 (1997).

[10] J. D. Kim, Y.U. Lee, and S. Kim, "IEICE Trans”, Fundamentals E86-A, vol. 859, (2003).

[11] J. D. Kim, J. Kim and G. Lee, "Efficient control system for PCR chips", Proceedings of SPIE 7929, (2011) February 14, San Francisco, USA.

[12] C. Y. Park, J. D. Kim, J. H. Ku, Y. S. Kim, H. J. Song, and J. W. Kim, "PCB-based PCR Chip", Sensor Letters, vol. 10, no. 1197, (2012).

[13] T. M. Hsieh, C. H. Luo, F.C. Huang, J. H. Wang, L. J. Chien and G. B. Lee, "Sensors and Actuators B 130", vol. 848, (2008).

[14] K. Shen, X. Chen, M. Guo and J. Cheng, "Sensors and Actuators B 105", vol. 251, (2005).

[15] K. Lians, S. O'Rourke, D. Sadler, M. Eliacin, C. Gamboa, R. Terbrueggen and M. Chason, "Sensors and Actuators B 138", vol. 21, (2009).

[16] P. C. Chen, D. S. Parka, B. H. You, N. Kim, T. Park, S. A. Soper, D. E. Nikitopoulos and M.C. Murphy, "Sensors and actuator B149", 291(2010).

[17] K. H. Chung, Y. H. Choi, and M. Y. Jung, "Natural Convenction PCR in a Disposable Polymer Chip", IEEE SENSORS Conference (2009).

[18] Q. Zout, Y. Miao, Y. Chen, U. Sridhar, C.S. Cheong, T. Chai, Y. Tie, C.H.L. The, T.M. Lim and C.K. Heng, "Sensors and Actuators A 102", 114 (2002).

[19] J. H. Wang, L. J. Chien, T. M. Hsieh, C. H. Luob, W. P. Chou, P. H. Chen, P. J. Chen, D.S. Lee and G. B. Lee, "Sensors and Actuators B 141", 329 (2009).

[20] D.N. Kim, Y. Lee and W.G. Koh, “Sensors and Actuators B 137”, 305 (2009).

[21]C. E. William, "User interface design for the engineer", Electro/94 International Conference Proceedings, Combined Volumes, 415 (1994).
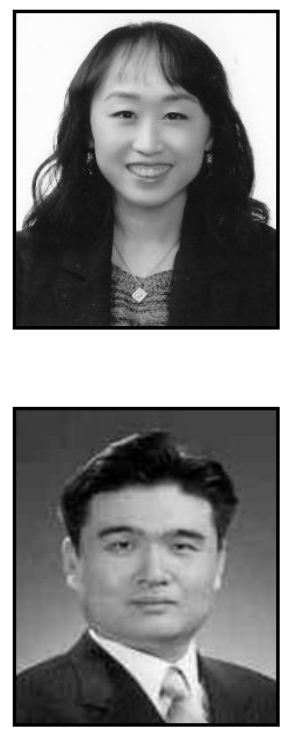

\section{Authors}

Ji-SooHwang, She received the B.S. and the M.S. in Computer Engineering from Hallym University. She is currently doing her doctoral studies in Computer Engineering at Hallym University. Her recent research focused on biomedical system and bioinformatics.

Yu-Seop Kim, He received the Ph.D. degree in Computer Engineering from Seoul National University. He is currently a Professor in the Department of Ubiquitous Computing at Hallym University, South Korea. His research interests are in the areas of bioinformatics, computational intelligence and natural language processing. 

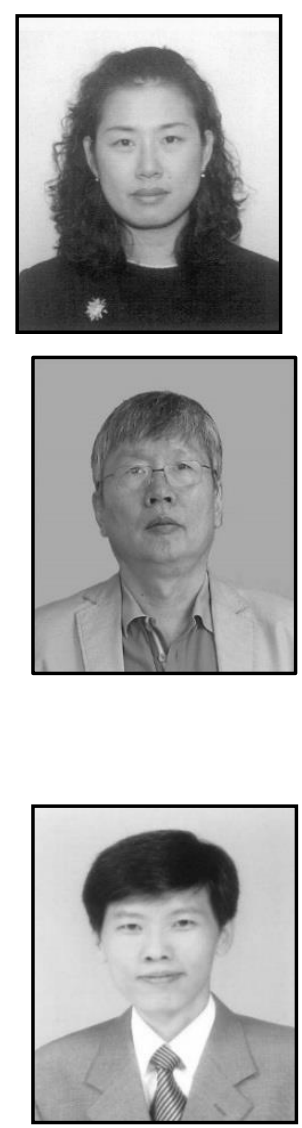

Hye-Jeong Song, She received the Ph.D. degree in Computer Engineering from Hallym University. She is a Professor in Department of Ubiquitous Computing, Hallym University. Her recent interests focus on biomedical system and bioinformatics

Jong-Dae Kim, He received the M.S. and the Ph.D. degrees in Electrical Engineering from Korea Advanced Institute of Science and Technology, Seoul, Korea, in 1984 and 1990, respectively. He worked for Samsung Electronics from 1988 to 2000 as an electrical engineer. He is a Professor in Department of Convergence Software, Hallym University. His recent interests focus on biomedical system and bioinformatics.

Chan-Young Park, He received the B.S. and the M.S. from Seoul National University and the Ph.D. degree from Korea Advanced Institute of Science and Technology in 1995. From 1991 to 1999, he worked at Samsung Electronics. He is currently a Professor in the Department of Ubiquitous Computing of Hallym University, Korea. His research interests are in Bio-IT convergence, Intelligent Transportation System and sensor networks. 